\title{
Les Oligochètes d'une rivière des Alpes du Sud, le Buech
}

\author{
A. Vedovini \\ P. Légier ${ }^{2}$ \\ F. Vespini ${ }^{2}$
}

Mots clés: Oligochaeta, Ecologie, Eaux courantes, Eutrophisation.

Dans le Buech, les communautés d'Oligochètes comprennent un nombre relativement élevé d'espèces, tandis que les densités des populations restent faibles. La fréquence relative des quatre familles présentes montre une dominance très nette des Naididae. Les agressions physiques (gravières) se traduisent par un appauvrissement quantitatif du peuplement, alors que les agressions chimiques (rejets d'eaux usées) ont l'effet contraire pour la famille des Naididae. La structure et la densité des communautés dans les stations soumises aux agressions chimiques montrent que celles-ci restent du niveau d'une simple eutrophisation.

\section{Oligochaetes of a river in the South Alps, the Buech}

Keywords : Oligochaeta, Ecology, Running waters, Eutrophisation.

In the River Buech, the oligochaete community has a relatively high number of species whereas the population density is quite low. The relative frequency of the four families present shows a marked dominance of the Naididae. The physical factors (gravels) are responsible for the low numbers in the population whereas the chemical factors (effluents of wastc water) have the opposite effect on the Naididae. The structure and density of the communities at the stations subjected to the chemical factors show that the latter remain at the level of a simple eutrophication.

\section{1. - Milieu et méthodes}

Le Buech est une rivière qui traverse ou longe trois départements, la Drôme, les Hautes-Alpes et les Alpes de Haute-Provence. Il résulte de la con. fluence de deux torrents qui prennent leur source aux environs de $2000 \mathrm{~m}$ d'altitude, le Grand Buech sur la commune de Lus-La-Croix-Haute et le Petit Buech vers Chaudon, au pied du Pic de l'Aiguille. La jonction des deux torrents a lieu au niveau de Serres, à $666 \mathrm{~m}$ d'altitude. Elle donne naissance au Buech proprement dit qui, après une quarantaine de kilomètres, se jette dans la Durance au niveau de Sisteron, à une altitude de $460 \mathrm{~m}$.

Le régime hydrologique du cours d'eau est de type nivopluvial ; il présente deux maxima différents en

1. Hydrobiologie, Universite de Provence, Centre Saint-Charles. 3 Place Victor Hugo, 13331 Marseille Cedex 3.

2. Biologie Animale Ecologie. Faculté des Sciences el Techuiques de Saint-Jérome, 13397 Marseille Cedex 13. amplitude. Le plus important se situe au printemps, au moment de la fonte des neiges. En 1983 la période des fortes eaux a débuté en mars avec un débit de $42.5 \mathrm{~m}^{3 /} \mathrm{s}$, et a atteint son maximumen mai avec 64 $\mathrm{m}^{3 / \mathrm{s}}$. Les variations sont brutales : le débit est passé de $32 \mathrm{a} 328 \mathrm{~m}^{3} / \mathrm{s}$ entre le 14 et le 16 mai, retombant à $88 \mathrm{~m}^{3 / s}$ trois jours plus tard. Durant l'étiage estival,le volume des eaux a varié entre 12 et $16 \mathrm{~m}^{3 /} / \mathrm{s}$. En novembre, de rares pluies ont provoqué une légère crue : le débit a été jaugé à 30 et $33 \mathrm{~m}^{3} / \mathrm{s}$ les 27 et 28 novembre à Laragne, contre $12 \mathrm{~m}^{3 /} / \mathrm{s}$ le 26 , mais il n'a pas dépassé une moyenne de $16 \mathrm{~m}^{3 / 5}$ pour le mois. Le déficit hydrique automnal a été sévère, et le volume des eaux véhiculées par le Buech est exceptionnellement faible pour cette saison.

Entre Serres et Sisteron, le cours d'eau est le siège d'agressions épisodiques liées aux activités agricoles de la vallée, comme les apports de nitrates, ainsi qu'à d'autres activités du secteur primaire, comme l'exploitation de gravières occasionnelles. Il s'y ajoute des pollutions chroniques, pollutions 
mécaniques liées au transport de matières solides en suspension en aval des gravières permanentes, et rejets d'eaux usées comme ceux de la ville de Laragne et de ses abattoirs. Sur ce tronçon de rivière, la pente du lit s'est considérablement atténuée par rapport à la partie haute où elle atteignait $10 \%$; elle n'est plus que de $0,75 \%$ entre Serres et Eyguians, et 0,50\% entre Eyguians et Sisteron. Physiographiquement, ce tronçon peut être assimilé à un rhithral sur les $3 / 4$ supérieurs, à un épipotamal en amont de Sisteron.

\section{1. - Les stations (fig.1).}

Cinq stations ont été prospectées entre Serres et Sisteron. Elles ont été choisies de façon à encadrer les deux sources majeures de pollution : pollutions mécaniques causées par une gravière, pollutions organiques dues aux rejets d'effluents urbains.

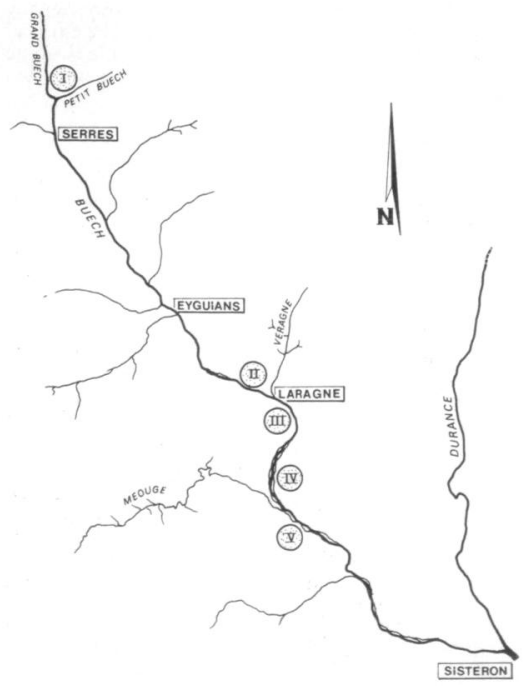

Fig. 1. Emplacement des stations sur le Buech.
La station $I$ a été choisie comme station de réf́rence, dans un secteur de forte productivité natu. relle mais peu soumis aux agressions liées aux aclivités humaines. Elle est prise sur le Grand Buech, aux environs de Serres, à quelques centaines dc mètres de sa confluence avec le Petit Buech. A ce niveau, la pente est de $0,75 \%$. La largeur du lit mineur varie entre 5 et $20 \mathrm{~m}$ selon les secteurs. La lame d'eau, d'une épaisseur de 20 à $30 \mathrm{~cm}$ en moyenne, se déplace à une vitesse de 60 à $80 \mathrm{~cm} / \mathrm{s}$ sur un substrat de galets lé plus souvent recouvert par un périphyton abondant essentiellement composé d'algues euryèces et d'une espèce d'eaux oligotrophes, la Chrysophycée Hydrurus foetidus. . Lal station comporte une ripisylve importante, les apports exogènes naturels en matière organique ne sont donc pas à négliger.

La station 2 est située $20 \mathrm{~km}$ plus bas, à environ $8 \mathrm{~km}$ en aval d'une gravière permanente. Le lit a une largeur d'une trentaine de mètres, la pente n'est plus que de $0,50 \%$. Les prélèvements sont réalisés au niveau d'un seuil de petits galets et de graviers grossiers, soumis à un courant souvent faible mais qui peut atteindre subitement 90 a $100 \mathrm{~cm} / \mathrm{s}$ au moment des crues. Ce seuil est situé à l'aval immédiat d'une mouille dont la profondeur dépasse $1,5 \mathrm{~m}$ même en période de basses eaux. Au niveau de cette station, le périphyton est très réduit. Il comporte des Chrysophycées, mais il est dominé par des Diatomées considérées comme mésosaprobes par Zelinka et Marvan (1961) et Sladecek (1973).

La station 3 se trouve à moins de $1 \mathrm{~km}$ de 1 a 2 . à $300 \mathrm{~m}$ seulement en aval de la confluence du Véragne, cours d'eau qui reçoit, sans épuration préalable, les eaux usées de la ville de Laragne et de ses abattoirs (le débit du Véragne a été jaugé à $1,3 \mathrm{~m}^{3 /} / \mathrm{s}$ le 24.1.83). Le lit mineur a 15 à $20 \mathrm{~m}$, le lit majeur environ $50 \mathrm{~m}$. Une lame d'eau de 30 à $50 \mathrm{~cm}$ court, rapidement par endroits ( 60 à $80 \mathrm{~cm} / \mathrm{s}$ ), plus lentement à d'autres, sur un substrat de gros galets et de blocs enduits d'un bioderme essentiellement dominé par des Cyanophycées Nostocales, des Euglénophycées et quelques Diatomées saprobiontes qui se développent en présence d'une pollution évidente.

La station 4 a été choisie à $3 \mathrm{~km}$ en aval des rejets, au niveau d'un élargissement de la vallée. Le lit principal, creusé dans des galets, peut se déplacer avec les crues. Il a habituellement une vingtaine de 
Tableau I. Analyses physicochimiques effectuées sur le Buech. Temp. : température ; Oxyd. : axydabilité.

\begin{tabular}{|c|c|c|c|c|c|c|c|c|}
\hline Mois & Station & $\underset{{ }^{\circ} \mathrm{C}}{\text { Temp }}$ & $\begin{array}{c}0_{2} \\
m g / l\end{array}$ & $\begin{array}{l}\mathrm{Cl}^{-} \\
\mathrm{mg} / \mathrm{l}\end{array}$ & $\begin{array}{l}\mathrm{NO}_{3}^{-} \\
\mathrm{mg} / \mathrm{l}\end{array}$ & $\begin{array}{c}\mathrm{PO}_{4}^{-}-- \\
\mathrm{mg} / \mathrm{l}\end{array}$ & $\begin{array}{c}\mathrm{DBO} 5 \\
\mathrm{mg} / \mathrm{l}\end{array}$ & $\begin{array}{c}\text { Oxyd } \\
\text { mg! }\end{array}$ \\
\hline & I & 3 & 13,9 & 7 & 1,12 & 0,01 & 2,01 & 0,65 \\
\hline & 2 & 0.6 & 14 & 17.5 & 2,44 & 0,01 & 2,22 & 0,75 \\
\hline \multirow[t]{5}{*}{01} & 3 & 2,9 & 13,8 & 18.5 & 2,12 & 0,045 & 2,22 & 0,5 \\
\hline & 4 & 2 & 14,1 & 18,5 & 2,38 & 0,04 & 2,22 & 0,8 \\
\hline & 5 & 1,5 & 13,6 & 16,5 & 2,16 & 0,015 & 2,42 & 0,55 \\
\hline & 1 & 13,2 & 10,6 & 7 & 1,82 & 0.01 & 2.62 & 0,52 \\
\hline & 2 & 13,5 & 10.5 & 9 & 1,45 & 0,01 & 2,82 & 0,57 \\
\hline \multirow[t]{5}{*}{03} & 3 & 13,8 & 10 & 9 & 1.45 & 0,01 & 2,72 & 1.22 \\
\hline & 4 & 9,6 & 11,5 & 9 & 1,62 & 0,01 & 3,02 & 0,62 \\
\hline & 5 & 8,9 & 11,6 & 8 & 1.52 & 0,01 & 3,12 & 0.7 \\
\hline & 1 & 14,4 & 11 & 7 & 1,25 & 0,01 & 0,9 & 0,85 \\
\hline & 2 & 18,3 & 10 & 24,5 & 2,15 & 0,01 & 1,31 & 0,7 \\
\hline \multirow[t]{5}{*}{06} & 3 & 19,6 & 10,3 & 24 & 2,22 & 0,32 & 1,51 & 0,95 \\
\hline & 4 & 21,1 & 10,3 & 24 & 2,32 & 0,014 & 2,01 & 0,8 \\
\hline & 5 & 21,6 & 9,9 & 20,5 & 2.04 & 0,01 & 0,6 & 0,85 \\
\hline & I & 19,2 & 9,4 & 10 & 2,95 & 0,027 & 3,02 & 0,6 \\
\hline & 2 & 20,8 & 8.7 & 85 & 3,65 & 0,017 & 3,73 & 0.55 \\
\hline \multirow[t]{5}{*}{08} & 3 & 19,9 & 7,4 & 80 & 3,46 & 0,188 & 3,83 & 1,9 \\
\hline & 4 & 19,5 & 9.5 & 52,5 & 4,25 & 0,018 & 2,21 & 0,65 \\
\hline & 5 & 20,3 & 8,9 & 55 & 3 & 0,012 & 2,82 & 0,5 \\
\hline & 1 & 12,8 & 11,3 & 22,5 & 2,32 & 0,01 & 1,11 & 0,40 \\
\hline & 2 & 21,3 & 9,6 & 65 & 2,16 & 0,01 & 1,21 & 0,77 \\
\hline \multirow[t]{5}{*}{09} & 3 & 19 & 10,5 & 85 & 2,12 & 0,250 & 3.43 & 1,07 \\
\hline & 4 & 19,3 & 11,9 & 80 & 2,32 & 0,115 & 2,32 & 0,75 \\
\hline & 5 & 19,2 & 9 & 57,5 & 2,36 & 0,01 & 1,41 & 0,52 \\
\hline & 1 & 6,1 & 14,7 & 25,5 & 1,07 & 0,03 & 1,1 & 0,55 \\
\hline & 2 & 6.3 & 12,6 & 57.5 & 1,53 & 0,01 & 3,33 & 0,42 \\
\hline \multirow[t]{3}{*}{11} & 3 & 6,5 & 12,3 & 55 & 1,55 & 0,171 & 5,14 & 0,57 \\
\hline & 4 & 7,5 & 12,8 & 60 & 1,69 & 0,105 & 4,44 & 0,6 \\
\hline & 5 & 8,1 & 13,1 & 45 & 1,31 & 0,023 & 3,53 & 0,47 \\
\hline
\end{tabular}

mètres de large pour une profondeur de 30 à $50 \mathrm{~cm}$. La vitesse du courant varie entre 50 et $70 \mathrm{~cm} / \mathrm{s}$. Le périphyton est assez semblable à celui de la station précédente, mais on y retrouve les Chrysophycées oligotrophes des stations 1 et 2 .

La station 5 est en amont de Ribiers, à $10 \mathrm{~km}$ environ de Laragne, à une centaine de mètres en aval de la confluence du torrent de la Méouge. La vallée est large et le lit mineur est assez difficile à définir. Le bras principal a habituellement une largeur de 20 à $30 \mathrm{~m}$ et une profondeur de 50 à $60 \mathrm{~cm}$. Le courant est toujours assez rapide ( 50 à $70 \mathrm{~cm} / \mathrm{s}$ ), sur un substrat de galets arrondis avec de rares blocs stabilisés. La communauté algale se caractérise par une diminution des Cyanophycées Nostocales.

\section{2. - Caractéristlques physico-chimiques.}

Les diverses analyses physicochimiques ont été regroupées dans le tableau I. Sur l'ensemble de son cours, le Buech présente des eaux calcaires et dures. Les valeurs relevées varient entre 150 et $225 \mathrm{mg}$ de $\mathrm{CaCO} 3 / \mathrm{l}$ pour la durée calcique et 180 et $225 \mathrm{mg}$ de $\mathrm{CaC03/1}$ pour la dureté totale. Les pH (de 7,6 à 8,1 ) traduisent des eaux faiblement ou moyennement alcalines. Les concentrations en bicarbonates (l'alcalinité correspondant aux alcalis libres et carbonates alcalins étant nulle) sont comprises entre 160 et $195 \mathrm{mg} / \mathrm{l}$ de $\mathrm{HCO}_{3}^{-}$Ces valeurs témoignent d'une alcalinité moyenne et se rapportent à des eaux très productives possédant un pouvoir tampon élevé (Nisbet et Vernaux, 1970). 
A l'image de la plupart des systèmes lotiques, les eaux du Buech se minéralisent progressivement de l'amont vers l'aval. A cette eutrophisation naturelle, se superposent, au niveau de la station 3 , les effets dus aux eaux résiduaires de la ville de Laragne. Les éléments traduisant une pollution domestique (orthophosphates, $\mathrm{NH}_{4}^{+}$, oxydabilité, DB05, carbone organique, azote organique particulaire, $\mathrm{Cl}^{-}$et conductivité) présentent des valeurs élevées dans le Véragne. L'autoépuration commence dans le secteur inférieur de l'affluent, où une partie importante de la charge est éliminée. Au niveau du Buech, le reste de la charge organique est largement dilué. Son impact est cependant visible à la station 3 où les valeurs des différents descripteurs abiotiques précédemment cités sont généralement supérieures à celles observées en 1 , et de manière plus at ténuée à la station 4 . A la station 5 par contre, les valeurs trouvées sont plus proches de celles de la station 1.

A cette évolution spatiale se superpose une évolution temporelle qui se traduit par une augmentation de la minéralisation dans toutes les stations entre le début de l'été et l'automne. L'évolution temporelle prime l'évolution spatiale. Ainsi, la station I présente au mois d'août un degré d'eutrophisation supérieur à celui de la 3 en janvier.

Les eaux sont toujours saturées ou sursaturées en oxygène, à l'exception d'un léger déficit observé en août à la station $3(83 \%)$, où au cours de la saison chaude l'apport de nutrients provoque un developpement bactérien et algal accru qui creuse encore l'écart des niveaux d'eutrophie observé entre cette station et les autres.

Dans un tel système, les phénomènes hydrauliques sont très importants, en particulier le phénomène de chasse que constituent les crues.

\section{3. - Méthodes d'échantillonnage.}

Les prélèvements ont été réalisés à l'aide d'un filet Surber (vide de maille $0,25 \mathrm{~mm}$ ) de $0.1 \mathrm{~m}^{2}$ de surface. Six prélèvements ont été effectués par station, trois dans des zones à courant rapide, trois dans des zones à courant lent (cependant, $d u$ fait de la rareté des fonds vaseux, le peuplement d'Oligochètes s'est révélé être le même dans les deux cas, et la distinction lénitique-lotique n'a pas été maintenue au niveau des résultats). Chaque station a fait l'objet de six campagnes de prélèvements pendant l'année
1983 (janvier, mars, juin, août, septembre et novembre), à l'exception de la 2 qui, du fait d'un accès difficile, n'a pu ètre visitée en mars et septembre. Le troisième prélèvement a été effectué en juin au lieu de mai à cause de la crue de printemps, à son maximum en mai ; par contre l'intervalle entre les prélèvements a été ramené à un mois en été, période la plus intéressante pour la faune.

\section{2. - Résultats}

\section{1. - Faunistique (Tableau II)}

Dix-huit espèces ont été récoltées : 10 Naididae, 6 Tubificidae, 2 Lumbriculidae. Les Enchytraeidae n'ont pas été déterminés.

\subsubsection{LUMBRICULIDAE}

Ils représentent $0,8 \%$ de tous les Oligochètes, ce qui constitue un pourcentage très faible pour un milieu, graviers et eau courante bien oxygénée, qui devrait leur être favorable. Des vers à màturité sexuelle ont été récoltés uniquement dans la station 1, ou Stylodrilus lemani parait être plus commune que Stylodrilus heringianus. Les immatures sont présents dans toutes les stations, mais avec une répartition très irrégulière. En fait seules les stations 1 et 3 ont des populations importantes, la station 1 regroupant $63 \%$ des captures et la $3,31 \%$.

Sur l'ensemble des stations, le nombre des captures est très faible en janvier et mars, et un premier pic de densité est observé en juin. En août le nombre de Lumbriculidae est pratiquement nul. En septembre on trouve un deuxième pic (densité maximum), les populations diminuant en novembre.

\subsubsection{TUBIFICIDAE}

Ils représentent $6,8 \%$ des captures, ce qui est également un pourcentage peu élevé. Quatre especes sont présentes de façon régulière : Limnodrilus hoffmeisteri ( $54,3 \%$ des effectifs de cette famille), Tubifex tubitex $(37,4 \%)$, L. claparedeanus $(6,5 \%)$ et Aulodrilus pluriseta $(1,1 \%)$. La répartition entre les stations est parallèle à celle des Lumbriculidae. Ils sont présents partout, mais les populations sont surtout importantes dans les stations 1 (67,3\% des Tubif $j$ cidae capturés) et $3(24,3 \%)$. 
Tableau II. Pourcentage moyen d'Oligochètes dans la communauté benthique. II. - Nombre moyen annuel de vers. $\left(/ \mathrm{m}^{2}\right)$. III. Abondance relative moyenne des différentes espèces

\begin{tabular}{|c|c|c|c|c|c|}
\hline Stations & 1 & 2 & 3 & 4 & 5 \\
\hline I & 5.45 & 3.44 & 20,3 & 10,9 & 3,44 \\
\hline Il & 1299 & 155 & 6441 & 2383 & 328 \\
\hline \multicolumn{6}{|l|}{ III } \\
\hline NAIDIDAE & & & & & \\
\hline Nais alpina & 44 & 6,9 & 1 & 15,9 & 37,1 \\
\hline Nais elinguis & 11,1 & 15,4 & 24,7 & 11,4 & 12,6 \\
\hline Nais communis & 0,5 & 6,1 & 13,2 & 4,8 & 2,8 \\
\hline Nais bretscheri & 0,7 & & 0,2 & & 3,5 \\
\hline Nais barbata & + & & 4,6 & 22,8 & 1,8 \\
\hline Nais pardalis & 1,1 & 4,5 & 1,4 & 5,5 & 12,6 \\
\hline Pristina idrensis & 14,1 & 31,8 & 42,5 & 41,2 & 22,4 \\
\hline Ophidonais serpentina & 0,6 & + & 5,8 & 6,3 & 1,6 \\
\hline Stylaria lacustris & 0,6 & & + & 3,9 & 0,4 \\
\hline Chaetogaster diaphanus & 2,2 & 2,4 & 2 & 2 & 0,3 \\
\hline \multicolumn{6}{|l|}{ TUBIFICIDAE } \\
\hline Tubitex tubifex & 14,5 & 11,4 & 1,6 & 0,6 & + \\
\hline Limnodrilus holfmeisteri & 3,2 & 16,4 & 1 & 0,4 & 1,2 \\
\hline Limnodrilus claparedeanus & 0,6 & 1,8 & 0,1 & + & + \\
\hline Aulodrilus pluriseta & 0,5 & 1,6 & + & + & + \\
\hline Peloscolex velutinus & + & & & & \\
\hline $\begin{array}{l}\text { Psammoryctides barbatus } \\
\text { LUMBRICULIDAE }\end{array}$ & & & & & + \\
\hline Stylodrilus heringianus & 1,9 & & 0,1 & + & + \\
\hline Stylodrilus lemani & 3,1 & & 0,2 & + & 0,4 \\
\hline \multicolumn{6}{|l|}{ ENCHYTRAEIDAE } \\
\hline Enchytraeidae g. spp. & 1,2 & 1,3 & 1,2 & 1 & 2,3 \\
\hline
\end{tabular}

Les deux espèces principales sont présentes toute l'année sous la forme adulte ; pour $L$. hoffmeisteri les effectifs les plus élevés se situent en automne et en hiver, pour $T$, tubifex en autornne.

\subsubsection{NAIDIDAE}

Avec $91,3 \%$ ils constituent le peuplement dominant de la faune des Oligochètes. Ils peuvent être subdivisés en deux contingents selon leur constance dans la communauté : ceux qui sont présents toute l'année et ceux qui apparaissent à la fin du printemps.

Quatre espèces ont été récoltées toute l'année. Ce sont Nais alpina, $N$. elinguis, $N$. communis et Ophidonais serpentina. $N$. alpina est une forme boréo alpine d'eau courante, sténotherme d'eau froide
(Giani 1976). Elle est dominante à la station 1, subsiste avec des effetifs réduits dans les stations 2,3 et 4, et redevient dominante à la 5. N. elinguis et $N$. communis se développent tout particulièrement dans la station 3 et, à un degré moindre, dans la 4. Dans la station 1, N. communis est peu abondante, ce qui est en contradiction avec les observations de Giani, qui la signale comme abondante dans les tor. rents pyrénéens en compagnie de $N$. alpina. La dernière espèce, $O$. serpentina, est présente partout, mais abondante surtout dans les stations 3 et 4 , à partir du mois d'août.

Les autres espèces n'ont été trouvées qu'à partir de juin. Ainsi $N$. bretscheri apparaît sporadiquement, dans les stations 1,3 et 5 . Ses effectifs restent toujours faibles. N. barbata, N. pardalis, Pristina idrensis 
et Chaetogaster diaphanus existent dans toutes les stations, avec des populations plus importantes en 3 et 4. Stylaria lacustris a été rencontrée en 1, 3, 4 et 5 ; ses populations sont très peu nombreuses. D'après Giani, ce serait d'ailleurs une espèce de plaine vivant essentiellement en eau stagnante.

\section{2. - Les stations}

Dans la station 1, station de référence, la densité du peuplement est basse (1 $300 \mathrm{vers} / \mathrm{m}^{2}$ en moyenne annuelle). Le peuplement est très faible en hiver (Tableau III). Il augmente fortement en juin puis retombe en août à un niveau hivernal. En septem. bre il at teint un niveau très supérieur à celui de juin, et redescend en novembre tout en demeurant élevé. Les Naididae constituent la famille dominante (Tableau IV), mais les Tubificidae sont aussi bien représentés. C'est la seule station où ont été récoltés des Lumbriculidae et des Enchytraeidae à maturité sexuelle. En ce qui concerne plus particulièrement les Naididae, $N$. alpina est l'espece dominante : deux autres sont bien représentées, $N$. elinguis et $P$. idrensis (fig. 2).

Tableau III. Evolution du nombre d'Oligochètes $\left(/ \mathrm{m}^{2}\right)$ dans les cing stations.

\begin{tabular}{rrrrrrr}
\hline Stations & 01 & 03 & 06 & 08 & 09 & 11 \\
\hline 1 & 278 & 136 & 2118 & 173 & 3913 & 1174 \\
2 & 28 & & 76 & 21 & & 492 \\
3 & 116 & 50 & 3968 & 1061 & 20315 & 13120 \\
4 & 2 & 91 & 1616 & 1011 & 5850 & 5735 \\
5 & 7 & 7 & 373 & 23 & 393 & 1163 \\
\hline
\end{tabular}

Tableau IV. Fréquences relative des quatre familles d'Oligochètes dans chaque station. $L$ :Lumbriculidae; $T$ : Tubificidae; $\mathrm{T}$ : Tubificidae ; N :Naididae ; $\mathrm{E}$ : Enchytraeidae.

\begin{tabular}{clrll}
\hline Stations & $\mathrm{L}$ & $\mathrm{T}$ & $\mathrm{N}$ & $\mathrm{E}$ \\
\hline $\mathbf{1}$ & 3,83 & 37,2 & $\mathbf{5 8 , 1}$ & 0,96 \\
$\mathbf{2}$ & $\mathbf{0 , 8}$ & $\mathbf{3 1 , 9}$ & $\mathbf{6 5 , 9}$ & 1,3 \\
$\mathbf{3}$ & 0,4 & 2,8 & $\mathbf{9 5 , 6}$ & 1,2 \\
$\mathbf{4}$ & 0,1 & 0,9 & 98,2 & 0,8 \\
5 & 0,6 & 1,9 & 95,2 & 2,3 \\
\hline
\end{tabular}

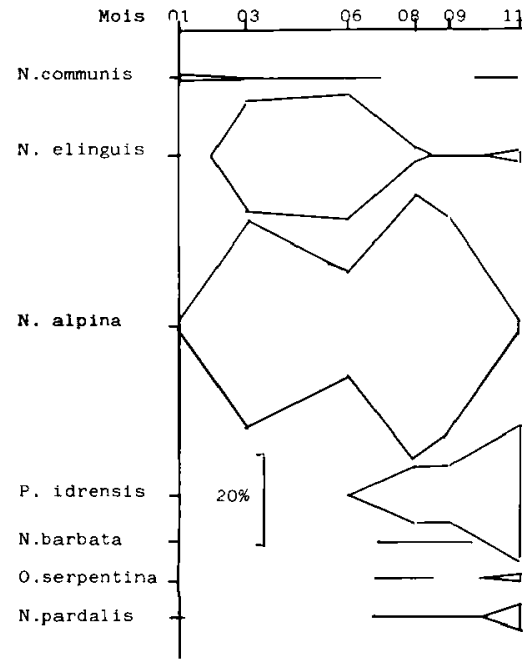

Fig.2. Evolution des populations des principales espèces de Naididae a la station 1.

La station 2 est beaucoup plus pauvre que la 1, le maximum du peuplement n'y atteignant pas $500 / \mathrm{m}^{2}$. La fréquence relative des farnilles est proche de celle trouvé dans la station 1 , avec une diminution du pourcentage des Lumbriculidae et des Tubificidae. Bien que les données soient peu nombreuses (pas de récolte en mars ni en septembre), $N$. elinguis semble être plus commune que $N$. alpina. La diminution générale de la densité du peuplement, la faible représentation des Lumbriculidae et la dominance probable de $N$. elinguis sur $N$. alpina traduisent l'influence de la pollution mécanique due à la gravière permanente installée en amont de cette station.

C'est la station 3 qui présente les plus fortes densités d'Oligochètes. Si en hiver les densités des populations sont inférieures à celles de la station 1 , en juin elles en atteignent le double. En aoùt la baisse est nette, le nombre de vers restant cependant 


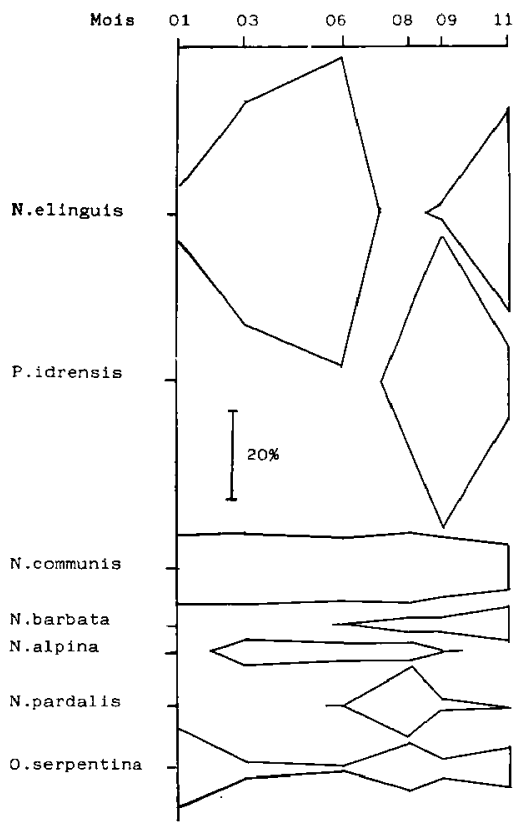

Fig. 3. Evolution des populations des principales espèces de Naididae à la station 3.

supérieur à celui de la station 1 . Il augmente ensuite considérablement en septembre, et diminue à nou. veau en novembre tout en restant très élevé. Ces effectifs importants sont dus aux Naididae, qui repésentent $95 \%$ des récoltes, les Tubificidae et les Lumbriculidae étant moins nombreux qu'en 1.N. alpina se maintient dans la station, mais elle est dominée par N. communis et surtout par $N$. elinguis (fig. 3). Parmi les espèces apparaissant en fin de printemps, on peut noter $N$. barbata et surtout Pristina idren. sis ; cette demière atteint en septembre 14000 individus au $\mathrm{m}^{2}$, représentant à elle seule pour ce mois $70 \%$ des Oligochètes de la station.

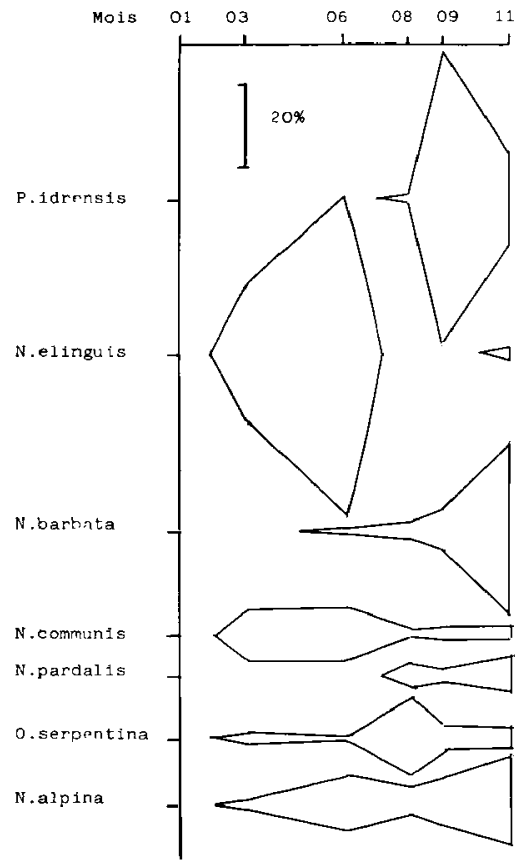

Fig.4. Evolution des populations des principales espèces de Naididae à la station 4.

A la station 4 , le nombre de vers récoltès est plus faible qu'à la 3 , mais il reste cependant très supérieur à celui de la 1. La proportion des Naididae est encore plus forte qu'à la 3 , cette augmentation se faisant au détriment des autres familles et surtout des Tubificidae. L'évolution annuelle est parallèle à celle de la station 3 : densités faibles en hiver, premier accroissement en juin, baisse en août, remontée à un niveau encore supérieur en fin d'année. Chez les Naididae (fig. 4) les effectifs de $N$. alpina et $N$. barbata s'accroissent, alors que ceux $N$. elinguis, $N$. communis et $P$. idrensis diminuent. 


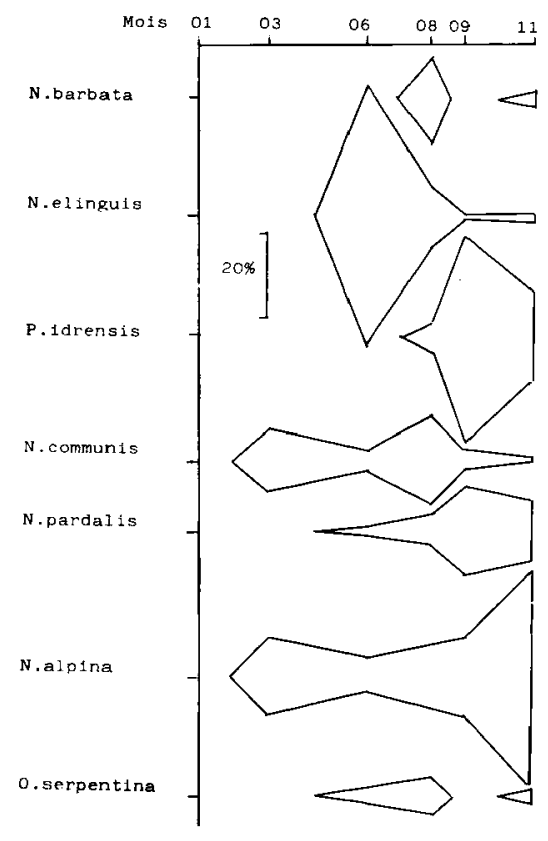

Fig.5. Evolution des populations des principales espèces de Naididae à la station 5 .

La communauté de vers de la station 5 est presque aussi pauvre que celle de la 2, la densité maximum n'y atteignant pas $1200 \mathrm{vers} / \mathrm{m}^{2}$. La répartition des familles est semblable à celle rencontrée dans les deux stations précédentes : très forte dominance des Naididae, peu de Lumbriculidae et de Tubificidae. On remarque toutefois une légère augmentation du nombre des Enchytraeidae. L'évolution annuelle suit sensiblement celle des stations 3 et 4 : densités très faibles en hiver, première augmentation en juin, baisse en août, remontée en fin d'année. Chez les Naididae, $N$. alpina redevient l'espèce dominante, comme dans la station 1 . Toutes les autres espèces sont présentes, mais avec des effectifs plus faibles. La faible densité du peuplement d'Oligochètes dans cette station reste inexpliquée. Tout au plus peut-on invoquer deux possibilités d'explication. D'une part les causes pourraient être physiques : changements du lit, grossièreté du substrat ou existence, en amont, de gravières occasionnelles. Mais d'autre part, la raison de la faible représentation des vers peut être recherchée dans le fonctionnement normal de l'écosystème. On a en effet noté, au niveau de la station 5 , la présence constante du Diptère Tipulidae Eriocera sp., prédateur d'oligochètes (et un accroissement numérique de sa population dans toutes les stations en août).

\section{3. - Discussion et conclusion}

Sur l'ensemble de la rivière, le nombre d'espèces est assez élevé (18), mais les densités des populations sont faibles (de quelques centaines à quelques milliers de vers au $\mathrm{m}^{2}$ ). Par suite de la grossièreté du substrat (comportant surtout des graviers, galets et blocs), les familles qui y vivent (Tubificidae, Lumbriculidae et Enchytraeidae) sont défavorisées et les Naididae dominent partout. Dans la station 1, prise comme station de référence, les Naididae représentent environ $58 \%$ de la communauté de vers, les Tubificidae $37 \%$ et les Lumbriculidae $4 \%$. Dans les quatre autres stations, les pourcentages des Tubificidae et des Lumbriculjdae diminuent au profit des Naididae.

L'évolution annuelle des communautés est parallèle dans toutes les stations. Les densités sont faibles en hiver, elles s'accroissent en juin, puis baissent fortement en août. En septembre elles sont à leur maximum et elles diminuent ensuite légèrement en novembre. La chute des densités en août est d'autant plus curieuse qu'on ne la retrouve pas chez les autres invertébrés de la rivière. Une explication possible est l'accroissement, à cette époque, et dans toutes les stations des populations d'un de leurs prédateurs, le Diptère Tipulidae Eriocera sp.

Dans les stations autres que la station 1 de réfé rence, les densités des populations varient en fonction des agressions subies par la rivière. Dans la 2 , et peut-être aussi la 5, elles diminuent du fait des 
pollutions mécaniques (gravières). Dans la 3 et à un moindre degré la 4, elles augmentent du fait du déversement des eaux usées de la ville de Laragne.

Dans ces deux dernières stations, on peut constater la prolifération de plusieurs especes de Naididae. Les deux plus abondantes sont $N$. elinguis $(6000$ vers par $\mathrm{m}^{2}$ en novembre à la 3 ) et $P$. idrensis ( 14000 vers par $\mathrm{m}^{2}$ en septembre à la 3 ). N. elinguis est bien connue pour sa polluorésistance (Brinkhurst 1965, Ladle 1971, Lafont 1977, Giani 1984). D'après Dumnika et Pasternak (1978) sa prolifération traduirait une pollution légère ou une eutrophisation. La polluorésistance de $P$. idrensis a aussi été signalée (Learner \& al 1971, Eyres \& al 1978, Giani 1984). En plus de ces deux espèces, on note un certain développement des populations de $N$. communis, dont la polluotolérance est également connue (Szczesny 1974, Lafont 1977, Giani 1984), de N. barbata, N. pardalis et $O$. serpentina.

Si l'impact des eaux usées de Laragne se fait nettement sentir au niveau de la station 3, il n'atteint cependant pas le niveau d'une pollution, mais d'une simple eutrophisation. A la station 3, aucune espèce n'est éliminée. Le pourcentage moyen d'oligochètes dans la communauté benthique de cette station s'élève à $20 \%$, mais on est bien loin des 60 à $80 \%$ qui caractérisent des conditions douteuses d'après Goodnight et Whitley (1960). Et la prolifération de $N$. elinguis et de $P$. idrensis n'a aucune commune mesure avec les 200000 vers au $\mathrm{m}^{2}$ signalés par Szczesny (1974) pour $N$. elinguis. Cet impact, encore bien ressenti a la station 4 , a disparu à la 5 , comme $l^{\prime}$ indiquent la structure du peuplement des Naididae ( $N$. alpina redevient l'espèce dominante) et J'accroissement du nombre des Enchytraeidae (Brink. hurst 1965).

Cette absence de discontinuité coénotique au niveau des déversements d'eaux usées s'observe aussi dans la distribution stationnelle des communautés d'insectes benthiques ou des algues épilithiques, essentiellement composées d'espèces euryèces.
La pression de la charge eutrophisante entraine seulement une modification de la structure numérique des communautés. En ce sens, dans le constat écologique réalisé sur la rivière Buech, les Naididae peuvent être considérés comme indicateurs quantitat ifs de changements survenant dans le budget énergétique de l'écosystème.

\section{Travaux cités}

Brinkhurst (R.O.). 1965. - Observations on the recovery of a British river from gross organic pollution. Hydrobiologia , 25; 9.51.

Dumnika (E.) \& Pasternak (K.). 1978. - The influence of physicochemical properties of water and bottom sediments in the river Nida on the distribution and numbers of Oligochaeta. Acta Hydrobiol. 20:215-232.

Eyres (J.P.), Williams (N.V.) \& Pugh- Thomas (M.). 1978. Ecological studies on Oligochaeta inhabiting depositing substrata in the Irwell, a polluted English river. Freshw, Biol. 8 : $25-32$.

Giani (N.). 1976. - Les Oligochetes aquatiques du sud-ouest de la France, Annls Limnol. 12: 107-125.

Giani (N.). 1984. - Le Riou Mort, affluent du Lot, pollué par métaux lourds, IV. Etude des Oligochètes. Annis Limnol. 20 : $167-181$.

Goodnight (C.J.) \& Whitley (L.S.). 1960. - Oligochaetes as indicators of pollution. Engng. Bull Purdue Univ, 45 : 139-142.

Ladle (M.). 1971. - The Biology of Oligochaeta from Dorset Chalk streams. Freshw. Biol. 1 : $87-97$.

Lafont (M.). 1977, - Les Oligochètes d'un cours d'eau montagnard pollué : le Bief Rouge. Annls Limnol. 13 : 157.167.

Learner (M.A.), Harcup (M.) \& Hughes (B.D.). 1971 - A survey of the macrofauna of the river Cynon, a polluted tributary of the river Taff (South Wales). Freshw. Biol. 1 : 339-368.

Nisbet (M.) \& Verneaux (J.). 1970. - Composantes chirniques des eaux courantes. Discussion et proposition de classes en tant que bases d'interprétation des analyses chimiques. AnnIs Lim. nol. 6 : 161-190.

Sladecek (V) 1973. System of water quality from the biological point of view. Ergeg. Limnol 7 : 1-218

Szczesny (B.). 1974. - The effect of sewage from the town of Krynica on the benthic invertebrate communities of the Kryniczarka stream. Acta Hydrobial. $16: 1-29$

Zelinka (M.) \& Marvan (P.). 1961. - Zur Präzisierung der biolo gischen Klassification der Reinheit fliessender Gewässer, $A r c h$. Hydrobiol. 57 : $389-407$ 